more than two months. As a last final attempt to obtain relief before having resort to an operation, which at the best could only be based on theory, she was taken into hospital and treated with bypodermic injections of pilocarpine. During these two weeks she had several attacks of vertigo. The examination of the ear gave the following results: Acoumeter in conchs. The voice was heard at two feet; a whisper was not heard. Rinne's test with tuning forks $\mathrm{C}$ and $\mathrm{O}^{2}$ gave negative results; $\mathrm{C}$. mastoid, -30 seconds. On testing with tuning forks $\mathrm{C}$ and lower notes were not heard. $\mathrm{C}^{1}$ was heard at 50 seconds; $\mathrm{C}^{2}$ at 40 seconds; $\mathrm{C}^{3}$ at 30 seconds; and $\mathrm{C}^{4} 2048$ at 25 seconds.

On Feb. 16th, 1904, she was anæsthetised and an ordinary radical mastoid operation was performed, with the exception that the innermost portion of the posterior wall was not removed, but the bony opening in the temporal bone was enlarged, forwards, upwards, and backwards. Anteriorly it was extended into the base of the zygomatic process of the temporal bone and postero-superiorly in such a way that the long diameter of the bony wound was from above downwards and forwards. The malleus and incus lying exposed after the removal of the external attic wall were removed. At this period of the operation the burr was substituted for the cutting gouge wbich had been previously employed. The next step in the operation consisted in exposing the upper and outer surfaces of the external semicircular canal in its whole extent. The antero-external portion of this canal was now followed forwards and inwards until the outer surface of the superior canal was brought into view. The whole of this canal was then removed by cutting it away with a medium-sized burr, leaving only the upper part of the arch or fornix untouched. The posterior rim of the external canal was then followed so as to bring into view the posterior canal which was burred away entirely. A large oval burr was now substituted for the medium-sized one previously used and the upper surface of the external-the only remaining canal-was cut away with the burr until the anterior half of the membranous canal was exposed. This was then removed with a small burr which was afterwards employed to make a medium-sized opening into the vestibule and an attempt was made to clean away the crista acustica at that end of the canal. This completed the operation, at which I was assisted by my colleague Mr. P. Macleod Yearsley. The wound was then swabbed out with Lister's strong solution, a precaution which I considered to be necessary to adopt on account of the impossibility of carrying out our operative procedures under strict antiseptic precautions. The external meatus was divided longitudinally through its posterior wall and the wound was packed and closed by the ordinary methods.

Immediately after the operation the patient suffered very severely from shock which lasted for about an hour. Slight chloroform sickness supervened but this was certainly hardly so much as is commonly the case. For the next 48 hours she lay coiled up on the right side, her thighs flexed on the abdomen and the legs on the thighs, in the position commonly described as that typical of cerebral irritation. During this period her eyes were tightly closed, but beneath the lids one could see that there were erratic movements of the eyeballs, and she resented any attempt to raise the lids, but though the light from the window fell directly on her face she preferred not to have the window darkened On the third day the wound was dressed and she would open her eyes if asked to do so but preferred to keep them shut. When they were opened the eyeballs were subject to irregular rhythmical movements both upwards and downwards and laterally. On the seventh day she sat up; on the tenth day she was able to walk with assistance for a few steps, and on the fourteenth day she could walk easily from the small room in which she was into that adjoining, a matter of some 15 to 20 yards there and back. She could, however, only turn towards the right or sound side. If she turned to the left she would have fallen over towards the right side. On the sixteenth day she walked downstairs with a little assistance and upstairs without any help. Her symptoms from that time lessened day by day. At the end of four weeks she was able to do everything without any fear of falling. Since the fourth day there had been no movements of the eyes, nor could any optic movements be induced by syringing the wound either with hot or cold lotions. It is now 14 weeks, or slightly over three months, since the operation. There has been no return of vertigo and the patient is enjoying better health than she has done for the ast few years.
There are a few points in respect to this case which are worth considering. The first is naturally the indication for operation. That can only be arrived at with anything approaching certainty when one has more than a single case to consider. Those cases which would appear to me to be the most suitable are those in which by a careful examination and after a course of treatment one has proved that the vertigo cannot be controlled, and at the same time that the deafness is sufficiently great to admit of no reasonable hope of alleviation, although, as will be seen when one comes to consider the question of tinnitus, the hearing does not appear to be diminished materially, and, indeed, the reverse was noticed in this case.

The operation itself is one in which the difficulties will be largely due to anatomical irregularities and to the age of the patient. In those skulls in which the middle fossa is at a lower level than the semicircular canals the operation would of necessity be more difficult and tedious. As to age, all patients excepting those who have passed middle age have a dense petrous bone, and the definition of the canals is extremely difficult. With regard to the symptoms following operation, it is impossible to avoid the conclusion that the extreme shock and irregular movements of the eyes were not entirely disconnected with the use of Lister's strong solution, for we are aware that irritation of the canals causes greater movement than simple section, and I would go so far as to say that strong and irritant antiseptics should not be employed. Finally, with regard to the tinnitus and the hearing power. The former was absolutely uninfluenced by the operation, that is to say her tinnitus is as bad as it was before. The hearing power, however, underwent a most extraordinary change. The voice, which had only been heard before the operation at a distance of two feet, was now well heard at five feet. Her bone conduction, which had previously been -30 seconds, was now -25 seconds, but whereas she had heard the $\mathrm{C}^{2}$ tuning fork, although very badly, before she was unable to detect it by air conduction afterwards.

\section{A CASE EXHIBITING THE ADAMS-STOKES SYNDROME. ${ }^{1}$}

By E. E. LASLETT, M.D. VICT., B.Sc. LOND., VISITING SURGEON TO THE HULI AND SCULCOATES DISPENSARY.

THE patient who is the subject of this paper is a thin, spare woman, aged 69 years, married, and has had nine children. When young she appears to have had several attacks of hæmatemesis and she has been liable to "bilious" attacks all her life. Apart from these she appears to have enjoyed very fair health and there is no history of rheumatic fever. She has a very large pendulous umbilical hernia, but this seems to have troubled her much less than one would expect, although its weight and position somewhat interfere with her walking. A year ago she had one prolonged fainting attack but otherwise seems to have been free from the ordinary symptoms of heart disease. The urine is light straw-coloured, with a specific gravity of 1010 and no albumin. Oscasionally she has to get up at night to pass urine, but this is not constant.

I was first called to see her on the evening of March 11th, 1904. She was lying on the floor semi-conscious, retching and vomiting small quantities of clear watery fluid, and moving her arms about in a wild, jerky way. The pulse was fairly full and slow. I did not count it. Her son told me that she had been much upset the day before by the breaking of an ornament-one of her few treasures I should fancy-and that earlier in the evening she had been much startled by a neighbour's sudden entrance and immediately sank down in a sort of faint. She soon recovered and, regarding it as a simple fainting attack, I advised her to rest in bed till I saw her again. During the early night she had three fainting. fits and again in the early part of the morning there was quite a number of attacks in rapid succession. Just after my arrival in the morning she had another seizure during which she was unconscious for about a minute with dilated pupils, grey cyanosis, stertorous breathing, rigidity of the body, and clonic irregular movements of the arms. Recovery of consciousness followed rapidly, indicated by cessation of the stertorous breathing, contraction of the

\footnotetext{
1 A paper read before the Hull Medical Society.
} 
pupils, a violent sneeze, and then she opened her eyes and spoke to those around. During the period of unconsciousness I noticed that the pulse at both wrists was quite absent. A minute or two later she had another seizure, apparently brought on like some of the others by the slight excitement of a neighbour's entrance. It was very short, but there was time to note that the pulse was again absent. Sneezing again heralded the return to consciousness. After this attack the pulse was 60 .

I saw her again in the early part of the afternoon. There had been several fits in the interval. The pulse was now from 40 to 42 per minute and slightly irregular in time. Sometimes there appeared to be a very small impulse given to the finger immediately after the normal pulse wave. Auscultation confirmed this, for after the second sound a feeble systolic sound was occasionally heard, not followed by a diastolic sound, a sign probably of what may be called an extra systole too feeble to reach the wrist. While I was listening to the heart I noticed that the beats became some. what rapidly slower and very feeble and almost immediately the respiration became deep and audible, the patient's head fell back, the pupils dilated, the body became rigid, and there was the same grey cyanosis with stertorous breathing. The noisy respiration so interfered with auscultation that $I$ was quite unable to satisfy myself as to whether the heart sounds were audible or not. All other signs of the heart beat had disappeared. At this moment her condition became so alarming that it did not seem permissible to continue the examination. A moment later breathing ceased, the jaw dropped, and she appeared to be dead, but a few seconds later the tongue moved, then the lips, the head turned, the pupils contracted, she sneezed, and immediately opened her eyes and spoke to me. The pulse had now risen to 60. At 9 o'clock in the evening the pulse was slow but slightly variable, the lowest being 36 . Generally it was 40 . It was not quite regular in time but with a very slow pulse it is not so easy to judge of the intervals as with a quick pulse. There had been five seizures since 1 o'clock. At this time the following was the condition of the heart. The sounds showed that the contractions exactly corresponded with the pulse. The sounds were of moderate intensity and there was a slight systolic murmur heard best towards the aortic area. The right side was dilated with a distinct impulse over the lower end of the sternum. The radial artery was tortuous and somewhat thickened. While I was further examining the pulse I noticed that the beats began to fail and I had time to warn her son that another attack was coming on. A second or two later the pulse failed in both wrists and a severe attack followed. For 45 seconds the pulse was absolutely imperceptible, then a feeble beat appeared, followed by another stronger and another, and consciousness returned. This "staircase effect" was very distinct owing to the slowness of the pulse and was obviously due to the gradual filling of the artery. After this attack the pulse remained slow, being 44 per minute.

During the three following days the patient had eight attacks in all. The pulse remained slow, it being from 48 to 50. On March 16th the pulse was 48. She had had two attacks during the night. On this day I examined the umbilical hernia. Probably this somewhat upset her, for on subsequently listening to the heart after a few moments the sounds became feeble and the beats slower and a typical attack followed, ushered in by a slight pallor of the face. As on a previous occasion the noisy respiration at first prevented my listening for the heart sounds, but as the stertor subsided and when the breathing was practically absent I found that for a period of 12 seconds the heart sounds were quite inaudible. Then feeble sounds appeared distinctly before the pulse reapceared the rate of the beat being at first rather rapid. During the attack the flickering in the jugular vein which at other times was very distinct quite disappeared. Consciousness returned slowly and it was a minute or two before she was able to answer questions. It was the worst and longest attack that I had seen and there was a good deal of mental depression for some little time after.

On March 22nd it was stated that there was a further attack on the afternoon of the 16th but that there had been none since. The pulse had been keeping at about 60 , occasionally rather slower. After this date the patient made good progress and was allowed to sit up for a short time on the $29 \mathrm{th}$, but I kept her recumbent for the most part during the next fortnight. The heart has much improved, the beats are quite regular, the systolic murmur is scarcely audible, and there is no impulse over the sternu $\mathrm{m}$. The pulse is generally about 60 . The treatment consis ted of rest, quiet, and medicinally three minims of liquor strychninæ and ten grains of potassium bromide three times daily, which she still continues to take.

There must have been about 30 seizures in all. There has never been pain before any of them. Usually they occurred without any warning but sometimes they were preceded by faintness or occasionally by a peculiar feeling as if someone were pressing on her chest and forcing her down. The urine was never passed during a fit. Consciousness as a rule returned very rapidly and this was the more striking because at the height of the attack the peculiar cyanosis, the widely dilated pupils, the dull cornea, the dropped jaw, and the cessation of breathing would lead almost anyone seeing the condition for the first time to say that the patient was dead.

Dr. Percy Kidd has recently published a clinical lecture on a case of Adams-Stokes disease." In this lecture he fully reviewed the literature and it is not therefore necessary for me to enter into this part of the subject except to mention one or two points which have a special bearing on the present case. Dr. Kidd's case is in many respects similar to $\mathrm{my}$ own. The patient was a woman, aged 58 years, whose pulse on admission varied from 25 to 28 . In two fits observed in the hospital the pulse was absent for 40 seconds and 90 seconds respectively. The heart was not examined during a fit. The patient improved and at the time of publication had not had an attack for over two months.

Apparently the complete absence of the pulse during the seizure had not been noted previously to Dr. Kidd's case. What is the actual condition of the heart during the fit? As previously stated on one occasion in my case the heart sounds were inaudible for 12 seconds, and it is not improbable that they would have been inandible almost from the beginning of the attack. On this subject Clifford Allbutt writes ${ }^{3}$ : "Very feeble heart beats may be inaudible even to the stethoscope. Fibrillary contraction is sometimes recovered from in animals, probably not in man." Several writers have suggested that the slowing of the heart in these cases is due to the action of the vagus inbibitory mechanism. We must suppose either that the inhibitory mechanism is in an abnormal condition of irritability or else that the heart muscle itself is for some reason peculiarly sensitive to its influence. It is important here to emphasise the very marked influence of emotion on the onset of the fits in my case as suggesting one way in which the inhibitory mechanism may be brought into play. If, now, the slowing of the heart in these cases be really due to vagus action it is not impossible that at a further stage the heart may be brought to an absolute standstill. So far as is known the simple action of the vagus cannot bring the heart to a final arrest. ${ }^{4}$ In prolonged experimental stimulation of the vagus after a time the heart "escapes" and resumes its beat. ${ }^{5}$ If, on the contrary, the cessation of the beat be due not to vagus inhibition but to intrinsic events everything almost is unfavourable for recovery. ${ }^{6}$ In any case it would be difficult to decide this interesting question, because the heart cannot be inspected as in an experiment upon an animal, but at least it is certain that an absolute stop for 12 seconds or more could be due only to the action of the vagus. Under any other condition recovery could not possibly take place after such a lengthy pause.

Hull.

2 The LanceT, Feb. 13th, 1904, p. 411.

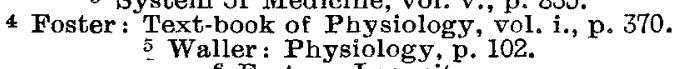

4 Foster: Text-book of Physiology, vol. i., p. 370.

Waller : Physiology, p. 102 . 6 Foster: Loc.

Britisi Mrdical Temperance Association.The twenty-eighth annual meeting of this association was held on May 26th at the London Temperance Hospital. Professor G. Sims Woodhead presided. The report showed that there had been 33 new members (abstaining registered practitioners) enrolled during the year and that there were now 568 members and 298 student associates. After the business meeting a conversazione was held at which short addresses were given by Professor Sims Woodhead and Mr. A. Pearce Gould, one of the vice-presidents. Mr. W. Chataway exhibited some magnificent natural colour photographs with the aid of an electric lantern. 